\title{
Evaluation of ground layer adaptive optics for the wide field optical spectrograph on the TMT
}

Jeff Stoesz, Jean-Pierre Véran, Scott Roberts, Brent Ellerbroek, Matthew Britton

Jeff Stoesz, Jean-Pierre Véran, Scott Roberts, Brent Ellerbroek, Matthew Britton, "Evaluation of ground layer adaptive optics for the wide field optical spectrograph on the TMT," Proc. SPIE 6272, Advances in Adaptive Optics II, 62724N (28 June 2006); doi: 10.1117/12.672578

SPIE Event: SPIE Astronomical Telescopes + Instrumentation, 2006, Orlando, Florida, United States 


\title{
Evaluation of Ground Layer Adaptive Optics for the Wide Field Optical Spectrograph on the TMT
}

\author{
Jeff Stoesz $^{a b}$, Jean-Pierre Véran ${ }^{a}$, Scott Roberts ${ }^{a}$, Brent Ellerbroek ${ }^{c}$, Matthew Britton $^{d}$ \\ ${ }^{a}$ National Research Council of Canada, Herzberg Institute of Astrophysics, 5071 W. Saanich \\ rd, Victoria, BC, V9E 2E7, Canada \\ ${ }^{b}$ Dept. of Physics and Astronomy, University of Victoria, P.O. Box 3055, Victoria, BC, V8W \\ 3P6, Canada \\ ${ }^{c}$ Thirty Meter Telescope Project, California Institute of Technology 1200 E. California Blvd. \\ Mail Code 102-8, Pasadena, CA 91125, USA \\ ${ }^{d}$ Caltech Optical Observatories, California Institute of Technology, 1200 E. California Blvd. \\ Mail Code 105-24, Pasadena, CA 91125, USA
}

\begin{abstract}
We arrive at a Ground Layer Adaptive Optics (GLAO) design that offers true seeing-improved performance and operation for the red and infrared wavelengths. The design requires an adaptive secondary (AM2) and that the sodium Laser Guide Star (LGS) launch telescope be able to steer four of the beams to 8.5 arcminutes off-axis. When provided with this, the proposed design is potentially the simplest, lowest cost design that can take the form of an upgrade. This is seen as a significant advantage over designs that would build an adaptive mirror into each of the four arms of WFOS. We show that the performance penalty for using one mirror instead of four to correct the entire 81 square arcminute WFOS field is minor.
\end{abstract}

Keywords: ground layer adaptive optics, astronomical instrumentation, extremely large telescope, wide-field spectroscopy

\section{INTRODUCTION}

The Thirty Meter Telescope (TMT) project identified a Wide Field Optical Spectrograph (WFOS) as a candidate first generation instrument. To further understand the costs and challenges of such an instrument a ten month feasibility study was undertaken for the project by Association of Canadian Universities for Research in Astronomy (ACURA), and the National Research Council Herzberg Institute of Astrophysics (NRC-HIA). Our design is built upon previous WFOS design studies done at NRC-HIA between November 2003 and November 2004. The overall instrument design and science motivation is described in [1] and references therein.

Curiosity about image improvement over large fields by correcting turbulence near the ground is not new [2] and still mostly unsatisfied due to a lack of data on the vertical distribution of the turbulence, especially in the bottom 1000 meters [3,4]. Our current understanding of the vertical distribution of turbulence at most sites does allow us to confidently say that a uniform correction of the $>75$ square arcminute field specified by the science cases for WFOS (see [1]) by a single conjugate adaptive optics system will be a modest improvement over a system that achieves the seeing limit. The gains for any of the designs we consider are best described as seeing improved and characterized by integration time savings. In this paper we evaluate the feasibility of several designs and arrive at a baseline which has a Ground Layer Adaptive Optics system correct the WFOS science field before entering the spectrograph optics [5].

In Section 2 we present our modeling tools, define performance metrics and predict average values for the baseline design in various observing conditions. In Section 3 we explore other possible architectures, affirming our chosen baseline design. Section 4 provides some detail on the main sub-systems for the baseline GLAO design and gives their top level requirements. Finally, Section 5 summarizes the important points from Section 3 and Section 4.

Send correspondence to jeff.stoesz@nrc-cnrc.gc.ca

Advances in Adaptive Optics II, edited by Brent L. Ellerbroek, Domenico Bonaccini Calia, Proc. of SPIE Vol. 6272, 62724N, (2006) · 0277-786X/06/\$15 · doi: 10.1117/12.672578 
Table 1. The probabilities for the nine Cerro Pachon profiles.

\begin{tabular}{c|ccc}
\hline \hline & \multicolumn{3}{|c}{ free atmosphere layers } \\
ground layers & good & typical & bad \\
\hline good & 0.0625 & 0.125 & 0.0625 \\
typical & 0.125 & 0.25 & 0.125 \\
bad & 0.0625 & 0.125 & 0.0625 \\
\hline
\end{tabular}

\section{PERFORMANCE ANALYSIS OF THE BASELINE DESIGN}

The analysis focuses on the fundamental error sources for the GLAO and no-GLAO cases. In the following subsections we describe the atmospheric input to the PSF model, the model itself, then the subsequent PSF analysis.

\subsection{The atmospheric database}

Accurate predictions for GLAO with the 81 square arcminute field of WFOS and AM2 conjugation require vertical resolution of better than 100 meters the turbulence strength $\left(C_{n}^{2}\right)$ profile at the ground, not to mention peculiar non-von Karman effects (e.g. from the telescope enclosure). In the meantime there is extensive, high quality MASS-DIMM data available at two candidate TMT sites (designated T1 and T2) plus high vertical resolution balloon data for Cerro Pachon.

The T1 and T2 profiles are defined with the ground layer at zero meters and the next highest layer at 500 meters. To account for the unknown scale height of the ground layer we also define a modified set of T1 and T2 profiles with the ground layer at 300 meters and are denoted $T 1_{300 m}$ and $T 2_{300 m}$. We model the AM2 cases with the DM conjugated to zero meters, which would make predictions based on the unmodified T1 and T2 profiles optimistic and the modified ones very pessimistic. These two sets will bracket the true predictions for an AM2 conjugated at a range of 300 meters.
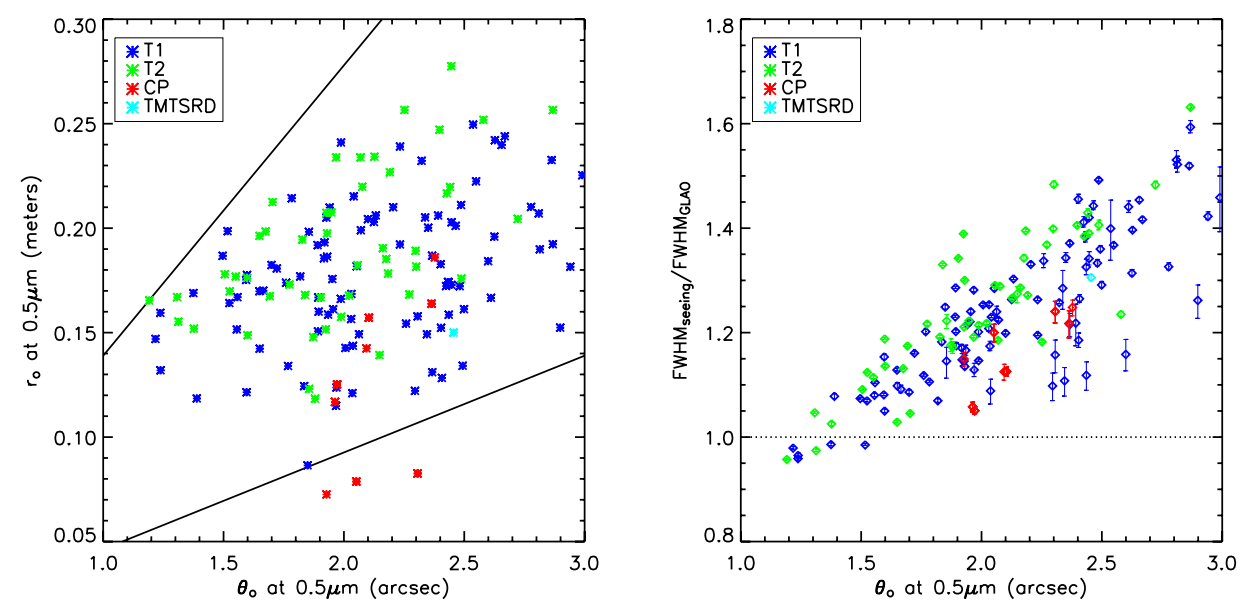

Figure 1 The left panel shows the $r_{o}$ and $\theta_{o}$ (at $\left.0.5 \mu m\right)$ for each profile. The two straight lines mark constant $\bar{h}=3 \mathrm{~km}, 9 \mathrm{~km}$ (lower, upper line; $\left.\bar{h}=0.314 r_{o} / \theta_{o}\right)$. The right panel shows the FWHM gain (at $\left.0.6 \mu m\right)$ of the baseline GLAO system versus $\theta_{o}($ at $0.5 \mu \mathrm{m})$. The diamonds correspond to the mean in the field of view for the AM2-5 baseline design. The vertical bars mark the minimum and maximum values in each field.

The Cerro Pachon (CP) balloon data were reduced to a nine profile model with the associated probabilities shown in Table 1. This CP model has been extensively used in other GLAO studies. For the readers who are 
familiar with them, the version of the profiles used here are the extended upper atmosphere version (i.e. four layers above $5 \mathrm{~km}$ ).

The solitary profile used in the TMT Science Requirements Document is plotted in Fig. 1 for reference. The Fried parameter $\left(r_{o}\right)$ and isoplanatic patch $\left(\theta_{o}\right)$ are moments of the $C_{n}^{2}$ profiles and admittedly do not capture all of the atmospheric character relevant to GLAO. Apropos, the $T 1_{300 m}$ and $T 2_{300 m}$ profiles are indistinguishable from the T1 and T2 profiles in the left panel of Fig. 1 The nine CP profiles (red asterisks) include three of the strongest seeing cases (smallest $r_{o}$ ) which correspond to the profiles with strong turbulence in the ground layers, also known as bad ground layer profiles.

It has been observed that the distribution of outer scale of turbulence at Mauna Kea is log-normal with a mean $\sim 30$ meters and FWHM $\sim 15$ meters. It was found that this distribution of outer scale does not noticeably bias the results in Section 3. Hence all three atmospheric scenarios $[\mathrm{T} 1, \mathrm{~T} 2, \mathrm{CP}]$ are simulated with a fixed outer scale $L_{o}=30$ meters.

\subsection{The GLAO PSF model}

The formalism used to simulate the Ground Layer AO (GLAO) Point Spread Function (PSF) for the analysis in the following sections is that of PAOLA, using a 2D FFT based algorithm by [6]. The PAOLA PSF model is coded in IDL and defined in the spatial frequency domain as a sum of Phase Spectra Densities (PSDs). There are four fundimental error sources in the PSD that are considered in this study.

$$
W(f)=W_{\text {aniso }}(\text { field location, } f)+W_{f i t}(f)+W_{\text {lag }}(\text { wind speed }, f)
$$

Where $f$ is the spatial frequency of the wavefront projected onto the primary mirror (called the pupil plane hereafter). For simplicity we choose a square actuator geometry with pitch $\Delta$ in the pupil plane so that the fitting error term $\left(W_{f i t}\right)$ for $f_{x}$ or $f_{y}>(2 \Delta)^{-1}$ is a von Karman spectrum (with $L_{o}$ specified in Section 2.1) and zero elsewhere. Similarly, the other terms are non-zero only where $f_{x}$ or $f_{y} \leq(2 \Delta)^{-1}$.

Anisoplanatism is of course a strong function of the field location of the PSF and is therefore important to LGS asterism trades. As shown in [7] anisoplanatism and fitting error are dominant over lag and WFS noise for the subaperture pitch of 0.59-1 meter considered in the asterism trades and we are therefore free to model with only anisoplanatism and fitting error.

The lag term is important to setting the requirement on the adaptive secondary mirror (AM2) and ultimately balances with the noise term through wavefront sensor (WFS) integration time, which is in turn dictated by the wind speed assuming frozen flow. Section 4.1 makes use of the lag term.

The PSD formulation used here is provided in [7] as it includes lag. In the case of no lag effects the this formulation reduces to that of [3] for beacons at finite range. LGS WFSs are blind to tip-tilt and focus (TTF) but his model does not include tip-tilt and focus (TTF) anisoplanatism. We therefore assume perfect TTF correction in each field using NGS WFSs. This implies a requirement on the patrol field of each NGS WFS needed to measure TTF, which is explained in Section 4.3.

The parameters used in the following sections are:

- DM height: at the ground

- sub-aperture pitch (subap spacing $\Delta)$ : 1 to 0.59 meters (30x30, 50x50)

- DM actuator pitch: matched to sub-apertures

- LGS asterism: various asterisms are used in Section 3 and are described in Table 2

- PSF locations: 10 locations for AM2-5 that take advantage of focal plane symmetry for field statistics (see Fig. 2). The spacing of sampled PSF locations is 1.125 arcminutes which well samples PSF variation for every case except Arm-1 (defined in Section 3) also known as Single-Layer GLAO (SLGLAO).

- Outer scale $\left(L_{o}\right): 30$ meters

- Zenith distance: zero only in Section 3

- Image wavelength: 0.6 and $0.8 \mu \mathrm{mm}$ in Section 3 

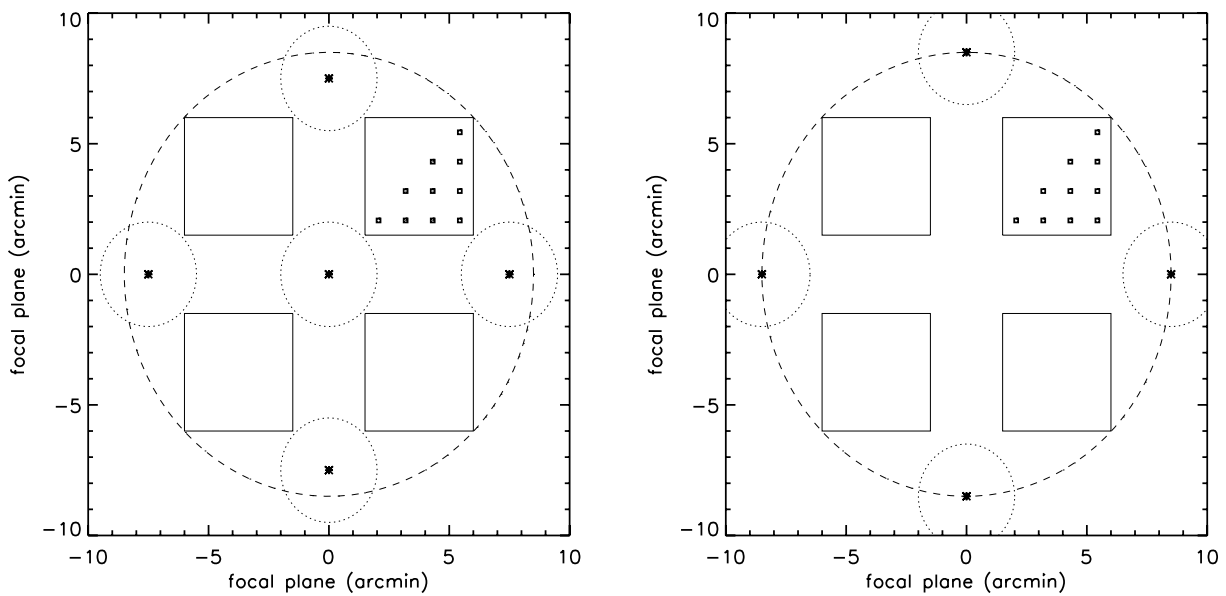

Figure 2 The field of view arrangement for the baseline AM2-5 configuration (left panel) and the compatible AM24-8.5 configuration (right panel). The asterisks are the LGS locations and the square points are the locations of the simulated PSFs for the field statistics presented in subsequent sections. It was determined that the AM2-5 design is incompatible with the opto-mechanical layout of the atmospheric dispersion compensators (ADCs) and the new baseline uses the LGS arrangement in the right panel.

\subsubsection{Image quality metrics}

There are five image quality metrics being used here to evaluate GLAO performance with WFOS: full width at half maximum (FWHM), width of 50\% ensquared energy (EE50), relative integration time (RIT) as well as integration time ratios for optimal apertures for imaging (ITR). Refer to [7] for the derivation and discussion of ITR.

In this study

$$
I T R=\frac{I T_{\text {seeing }}(\bar{\omega})}{I T(\bar{\omega})}
$$

where $I T$ is the integration time on the PSF being considered and $I T_{\text {seeing }}$ is the integration time for the noGLAO PSF to achieve the same signal to noise ratio. We consider only background noise limited point sources, which determines $\bar{\omega}$, the optimal aperture. The relative integration time is then

$$
R I T=\frac{\bar{\omega}^{2}}{\left((E E(\bar{\omega}))^{2}\right.} .
$$

See [7] for further detail. 
Table 2. Summary of GLAO architectures considered in the study.

\begin{tabular}{ccc}
\hline \hline Architecture name & DM location & LGS asterism \\
\hline AM2-4 & Adaptive Secondary Mirror & 4 LGSs, one centred on each science field \\
AM2-4-8.5 & Adaptive Secondary Mirror & 4 LGSs outside of the WFOS science fields \\
AM2-5 & Adaptive Secondary Mirror & 5 LGSs outside of the WFOS science fields \\
AM2-8 & Adaptive Secondary Mirror & 8 LGSs bordering on the WFOS science fields \\
Arm-1 & Within modified WFOS Collimator & 1 LGS centered on each science field (4 total) \\
Arm-2 & Within modified WFOS Collimator & 2 LGSs bordering on each science field (8 total) \\
AM2-..* & Adaptive Secondary Mirror & $\ldots$ LGSs outside of the WFOS science fields \\
Arm- $4^{*}$ & Within modified WFOS Collimator & 4 LGSs bordering on each science field (12 total) \\
\hline
\end{tabular}

${ }^{*}$ These architectures have more than the expected number of LGSs to be provided by the LGS facility and are included for illustrative purposes.

\section{ARCHITECTURE TRADES}

Summarized in Table 2 are eight GLAO architectures that cover the range of considered LGS constellations and deformable mirror (DM) implementations. The focal plane detail for AM2-5 and AM2-4 are shown in Fig. 2.

The first three options in Table 2 have the telescope secondary mirror (AM2) as the DM, common to the paths of all four WFOS subfields. The Arm configurations (Arm-1, Arm-2, and Arm-4) use a DM in the WFOS collimator in each of the four barrels. The current WFOS design does not provide an intermediate pupil location for a DM within the collimator. In order to provide this location we expect that four additional reflections would be required (including the DM). With an average surface reflectivity of 0.97 , this is a $\sim 10 \%$ loss of throughput. A rough order of magnitude estimate for implementing this collimator / DM combination is $\$ 2.5 \mathrm{M}$ per arm.

The upper bound on the number of LGSs for WFOS-GLAO will be the number planned for other TMT AO systems. Therefore, based on current planning for NFIRAOS, we expect to have seven to nine LGSs available. A requirement from WFOS-GLAO would be that the LGSs be able to move out to edges of the WFOS field (17 arcminutes diameter).

For the Arm-1 and AM2-4 options an LGS is projected within each WFOS science field. LGSs within the science field could be picked off with mirrors close to the focal plane or by placing dichroic beamsplitter windows in front of the field. The beamsplitter option is a technical challenge because of the size of the substrate required, and the difficulty in applying a multi-layer coating that will provide a notch filter for $589 \mathrm{~nm}$ light. Also, with this option there would be a loss of throughput of science light across the full wavelength range. 


\subsection{Simulation results}

In this section we will show how the PSF is affected by the AM2 vs. Arm architectures, LGS asterism, and actuator density.

\subsubsection{Arm vs. AM2}

The AM2-4-8.5 is the chosen baseline design and offers performance very similar to AM2-5. Here we compare AM2-4-8.5 to Arm-4 with the $T 1_{300 m}$ and $T 2_{300 m}$ scenarios, which ensures that we will not underestimate the performance of potential of the Arm architectures. The $T 1_{300 m}$ and $T 2_{300 m}$ are the worst scenarios for the AM2 case, with the nearest layer to the DM at 300 meters away and the next nearest at 500 meters away, the greatest amount of turbulence is in the GLAO gray zone (described in [3]). The left panel of Fig. 3 shows the asterism for both AM2-4-8.5 and Arm-4. To achieve the latter requires at least 12 LGS and vignetting of the science field.

The cumulative histogram results for ITR (right panel Fig. 3) show that changing from the baseline design to the powerful Arm- 4 design would change the median ITR at $0.6 \mu m$ from 1.18 to 1.3 half the time. Both cases here are simulated with a pitch of 1 meter. The Arm- 4 case with 0.59 meter pitch (50x50 on each DM) achieves a slight better 1.32 half the time.
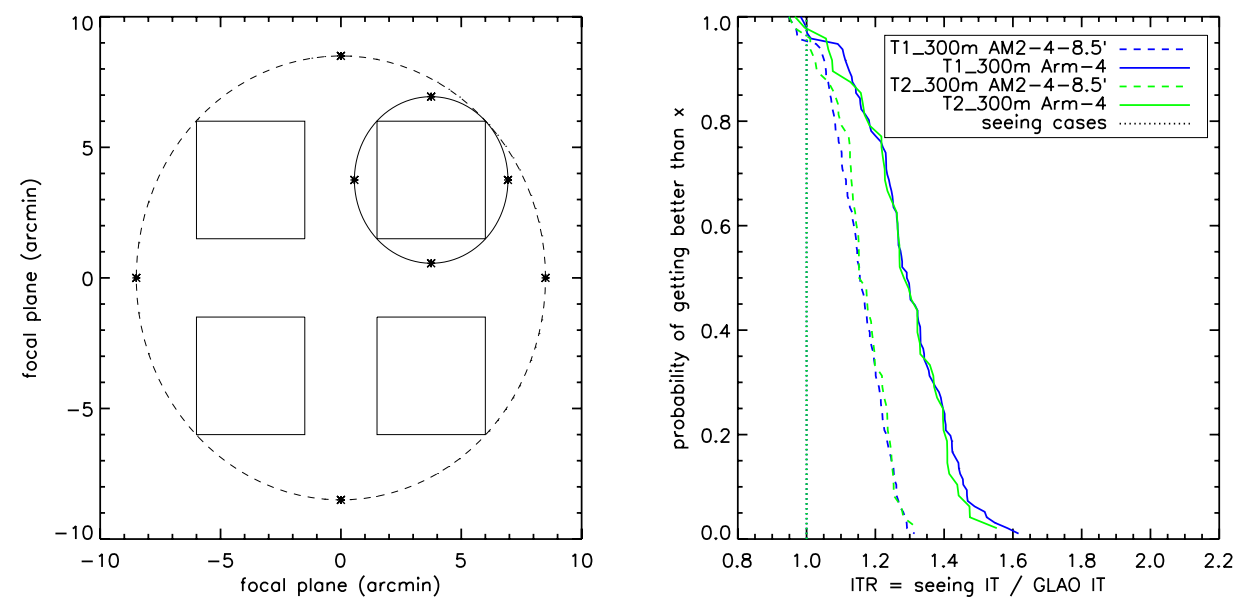

Figure 3 Comparison of Arm-4 and AM2-4-8.5. Left panel: is the WFOS focal plane with the guide stars of the Arm- 4 case connected by the circle drawn with a solid line of diameter 6.38 arcminutes. The baseline AM2-4-8.5 guide stars are connected by the dashed line, 17 arcminutes in diameter. Right panel: performance show with solid and dashed lines in a of cumulative histogram of ITR.

\subsubsection{AM2 variations}

We continue the trade study with the Cerro Pachon atmospheric scenario because it offers proper vertical resolution of $C_{n}^{2}$ at $0,20,50$, and 100 meters. The left panel of Fig. 4 shows cumulative histograms of relative integration time (RIT) at 0.8 microns. It shows RIT improving slightly as one goes from a 30x30 to a 50x50 AM2 system (pitch from 1 to 0.59 meters). The biggest improvement occurs where the GLAO DM has the greatest potential, strong turbulence in the lower layers of these $\mathrm{CP}$ profiles.

The right panel of Fig. 4 shows that, with a 50x50 system, any number of guide stars greater than or equal to four gives roughly the same performance. Hence four or five LGS are sufficient to command a 50x50 system and therefore a 30x30. As in the left panel, the order of the list of cases in the legend from left to right matches the order of solid curves. Arm-1 and AM2-4 are not included in these plots as the field mean is similar to the others yet the standard deviation is too large to appear in Fig. 5. 

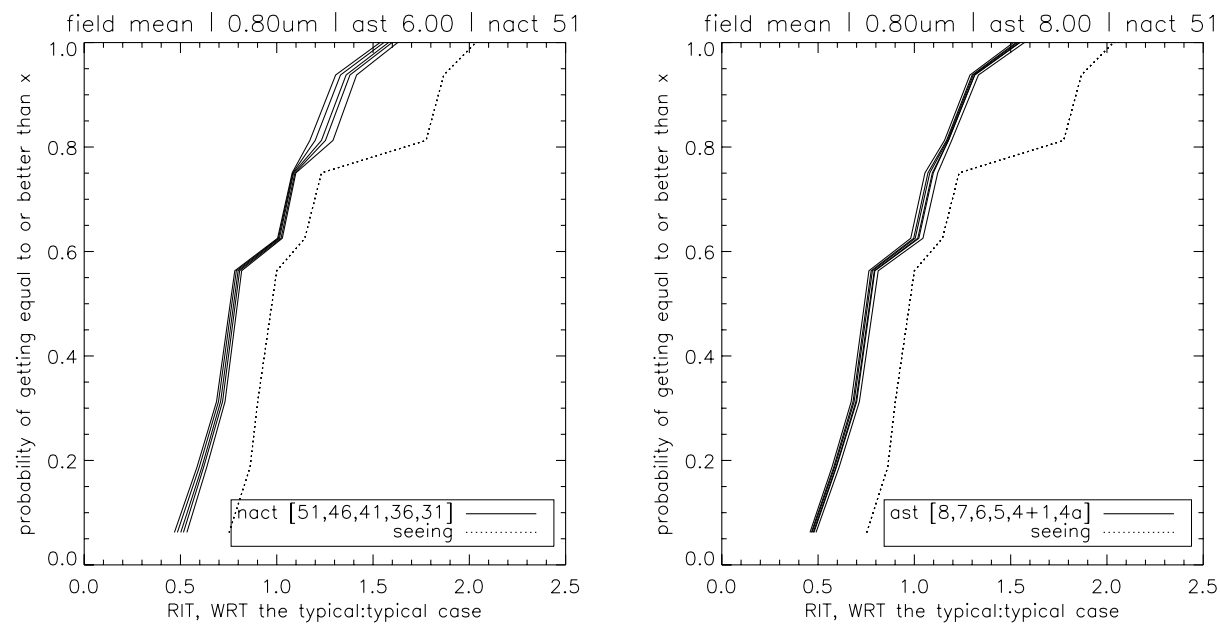

Figure 4 The left panel is for a hexagon-like asterism at various actuator densities indicated in the legend by the number of actuators across the diameter of the pupil. The right panel are the results with 50x50 actuators and various ring asterisms indicated in the legend by the number of LGS in the ring, with or without an extra added in center ("+1").
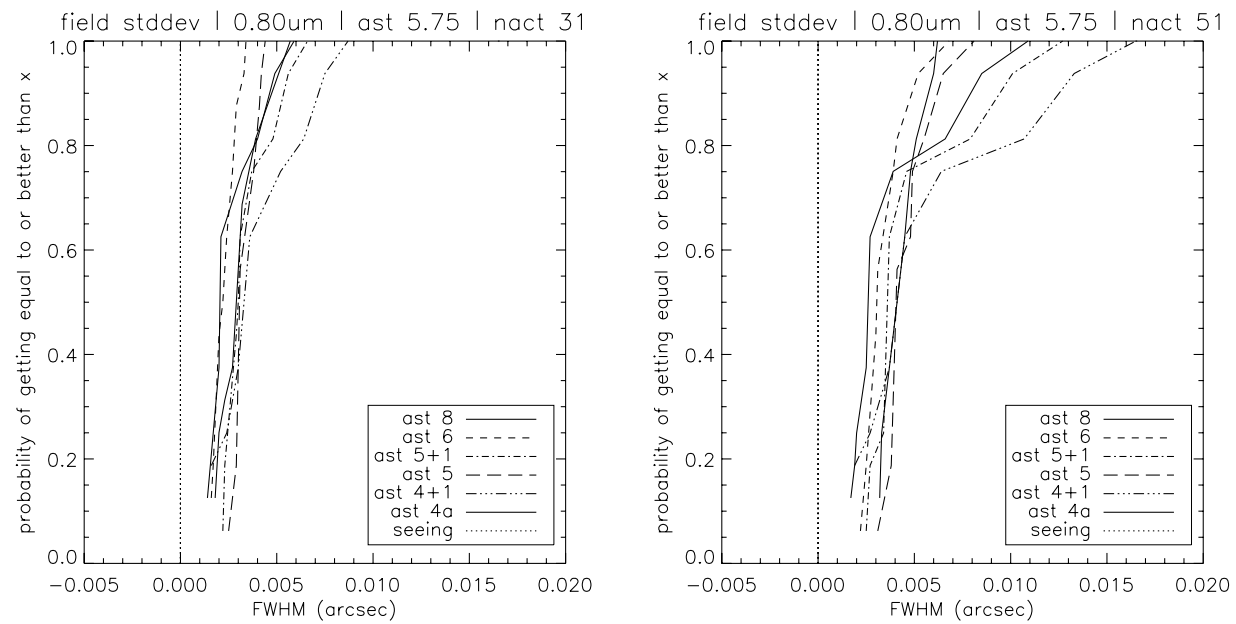

Figure 5 Cumulative histograms of field standard deviation rather than mean of FWHM. Here we see that all of the +1 asterisms (dash-dotted curves) produce much more PSF nonuniformity when the low altitude turbulence is strong. The only thing distinguishing the +1 asterism from the non +1 with the same total number of guide stars is the increased nonuniformity. 
Table 3. Summary of architecture cost and 50th percentile performance at zenith and $0.8 \mu m$.

\begin{tabular}{cccccc}
\hline \hline Architecture & $\begin{array}{c}\text { ITR, field mean/median } \\
100 \% \text { TP }\end{array}$ & ITR, field std.dev. & $\begin{array}{c}\text { First light } \\
\text { or upgrade }\end{array}$ & $\begin{array}{c}\text { Vignetted fraction } \\
\text { of 4.5'x4.5' field }\end{array}$ & $\begin{array}{c}\text { Estimated } \\
\text { cost (\$USD) }\end{array}$ \\
\hline AM2-4-8.5 & $1.20^{*} / 1.20^{*}$ & $0.009^{*}$ & upgrade & None & $\$ 2.2 \mathrm{M}$ \\
AM2-5 & $1.21 / 1.22$ & 0.012 & upgrade & None & $\$ 2.5 \mathrm{M}$ \\
Arm-4 & $1.45^{*} / 1.45^{*}$ & $0.007^{*}$ & not feasible & $2.0 \%$ & $>\$ 12.6 \mathrm{M}$ \\
AM2-4 & $1.23 / 1.22$ & 0.05 & upgrade & $6.6 \%$ & $\$ 2.2 \mathrm{M}$ \\
AM2-8 & $1.24 / 1.24$ & 0.016 & upgrade & $3.0 \%$ & $\$ 3.4 \mathrm{M}$ \\
Arm-1 & $1.23 / 1.10$ & 0.41 & first light & $6.6 \%$ & $\$ 10.2 \mathrm{M}$ \\
Arm-2 & $1.22 / 1.21$ & 0.09 & first light & $3.0 \%$ & $\$ 11.4 \mathrm{M}$ \\
\hline
\end{tabular}

${ }^{*}$ These values are interpolated from results at 0.6 and $1.25 \mu \mathrm{m}$

Table 4. Rough cost estimates for various items in (\$USD).

\begin{tabular}{lc}
\hline \hline \multicolumn{1}{c}{ item } & cost \\
\hline Adaptive secondary - provided for other instruments & $\$ 0 \mathrm{M}$ \\
NGS WFS - provided for WFOS without GLAO & $\$ 0 \mathrm{M}$ \\
LGS beacons - provided for other instruments & $\$ 0 \mathrm{M}$ \\
LGS WFS - per unit & $\$ 0.3 \mathrm{M}$ \\
Arm Collimator - per arm - DM $\$ 1 \mathrm{M}(\sim \$ 1.25 \mathrm{k}$ per actuator $)-3$ Mirrors $\$ 1 \mathrm{M}$ & $\$ 2.0 \mathrm{M}$ \\
Other costs & $\$ 1 \mathrm{M}$ \\
\hline
\end{tabular}

\subsection{Architecture, performance, cost and the baseline design}

Table 3 summarizes the characteristics of each of the systems. Image quality is assessed using ITR. The ITR values quoted in the table are the field mean and median values. The PSF non-uniformity is represented by the field standard deviation of the ITR values. The vignetting value is the percent of the field obscured by the LGS WFS pickoff mirrors. Note that these are a lower bound on the actual vignetting, since they only consider the minimum size of mirror required, and do not consider mechanical elements required to support the mirrors. The cost estimation is detailed in Table 4.

Among the Arm designs only Arm-4 is competitive. Arm- 1 and Arm-2 also demand four single conjugate DMs that may rival the cost and complexity of AM2. When an LGS is placed in the science field there is higher non-uniformity of the correction, in addition to the problem of gathering the light with vignetting pick-off mirrors or impossible beamsplitters. Having an LGS in or near the field also does not appreciably increase the field mean ITR. For these reasons we eliminate Arm-1 and Arm-2.

AM2-4 is also not preferred as it also suffers from PSF non-uniformity and vignetting.

AM2-8 and Arm-4 demand eight and twelve LGS beacons, the latter also requiring four single conjugate DMs that may rival the cost and complexity of AM2.

AM2-5 was previously chosen as the baseline design, but AM2-4-8.5 is preferred as it provides essentially the same field mean ITR, but with more uniformity.

The simulations in Table 3 used the CP atmospheric scenario with the DM conjugated to zero meters in every case, which is well conjugated with the turbulence. AM2 is a Gegorian secondary and will in fact be conjugated to +300 meters and for the 1 meter pitch and 17 arcminute field of view simulated here the gradual gray zone transition (see Ref. [3]) is around 200 meters from the DM and thus has an impact on accurate performance predictions, but does not impact the differential performance used for the trade studies. 
The possibility of the AM2 upgrade for other TMT adaptive optics systems makes AM2 very attractive as the primary corrector for WFOS-GLAO. The Arm configurations requires more initial investment and risk in WFOS for an ITR of 1.45 rather than 1.20 with AM2 (at $0.8 \mu m)$. The risk and cost trade-off for Arm is not feasible.

\section{BASELINE SYSTEM DESIGN}

In the previous sections we evaluated the performance of GLAO and performed basic system trades. In this section, we examine each of the sub-systems and their requirements.

\subsection{Deformable mirror}

The baseline design will use an adaptive secondary mirror (AM2) for wave-front correction. All the other TMT AO systems would use AM2 only as a woofer, which relaxes their requirements. It is therefore important to make sure that the AM2 woofer will be suitable as the primary corrector for GLAO. A feasibility study for AM2 is currently being carried out by Sagem. Our assumptions on the characteristics of AM2 are based on their preliminary results.

\subsubsection{Actuator density}

The expected inter-actuator spacing on AM2 itself is 6 to $8 \mathrm{~cm}$ across a mirror $\sim 3.5$ meters. This works out to be between 43 and 58 actuators across the pupil diameter. The actuators will be arranged in a hexagonal pattern. One should remember however that it may be not possible to apply some high spatial frequency modes to AM2, because too much force would be required. Hence AM2 will be equivalent to an unconstrained DM with a lower actuator density. It is reasonable to assume that the AM2 woofer will perform at least as well as an unconstrained DM with 30x30 rectangular grid and will perform somewhat worse than a 50x50 DM, which were simulated in Section 3.1.2.

\subsubsection{Temporal bandwidth}

Based on the AM2 design study by Sagem we expect to achieve a temporal bandwidth for AM2 between 100 and $1000 \mathrm{~Hz}$ (-3 dB bandwidth). AM2 will be slower than a normal piezo-based DM (which can be assumed perfect) and thus it is interesting to investigate the effect on delivered image quality. Our analytical modeling of GLAO does not allow us to input any dynamic properties of the DM, which is assumed to be perfect. It does allow us to change the time delay in the AO loop.

For the pathological case we choose a severely strong wind model with 20 meter per second wind below 2000 meters and $5 C_{n}^{2}$ profiles from the T1 database selected for strong turbulence below 2000 meters and weak turbulence above. We find that with a pitch of 1 meter, $500 \mathrm{~Hz}$ frame rate and a 10 milliseconds AM2 delay results in noticeably degraded ITR for these profiles. With a $3 \mathrm{~ms}$ AM2 delay there was no performance degradation and therefore conclude that an effective delay of $3 \mathrm{~ms}$ or less will have no impact on GLAO performance.

\subsection{LGS WFS}

\subsubsection{Radial format CCDs}

The baseline configuration for WFOS-GLAO is to use four or five LGS WFSs. A well known problem with LGS wavefront sensing on a 30 metre telescope is that off-axis sub-apertures see the thickness of the sodium layer and produce a radially elongated spot: at the centre of the pupil, the spot is typically circular and has an FWHM of $\sim 1$ arcsec. At the edge of the pupil, the size of the spot is still $\sim 1 \operatorname{arcsec}$ in the tangential direction, but is $\sim 4$ arcsec in the radial direction. An elongated spot produces a noisier measurement and to mitigate this effect, we plan to use CCDs with a radial geometry and special clocking. They are being developed and are the baseline for the Narrow Field Infra-Red Adaptive Optics System (NFIRAOS) for TMT. In each sub-aperture the CCD has a rectangular pixelated area (e.g. 12x6) which has a radial orientation that puts the long side of the rectangle parallel to the direction of the spot elongation. 
Table 5. Amount of static astigmatism and coma aberrations that will degrade the image of an LGS at 7.5 arcmin off-axis, for different range distances, in micron RMS of wave-front error. This number is converted in arcsec of WFS spot displacement, for a spot at the edge of the telescope pupil.

\begin{tabular}{ccc}
\hline \hline & Astigmatism & Coma \\
\hline $\mathrm{Z}=85 \mathrm{~km}$ & $-2.40 \mu \mathrm{m}$ RMS & $-1.78 \mu \mathrm{m}$ RMS \\
& -0.16 arcsec & -0.48 arcsec \\
$\mathrm{Z}=150 \mathrm{~km}$ & $-2.39 \mu m \mathrm{RMS}$ & $-1.04 \mu \mathrm{m} \mathrm{RMS}$ \\
& $-0.16 \operatorname{arcsec}$ & $-0.28 \operatorname{arcsec}$ \\
$\mathrm{Z}=85 \mathrm{~km}-\mathrm{Z}=150 \mathrm{~km}$ & $-0.01 \mu m \mathrm{RMS}$ & $-0.74 \mu \mathrm{m}$ RMS \\
& $0.00 \operatorname{arcsec}$ & $-0.2 \operatorname{arcsec}$ \\
\hline
\end{tabular}

\subsubsection{Non-common path aberrations}

TMT is currently an aplanatic Gregorian telescope and therefore produces anastigmatic off-axis images. For the same reason, the image of an off-axis source not at infinity, such as an LGS, will also be degraded by coma, which is a function of the range distance. In our baseline configuration, four LGSs lie at 7.5 arcmin off-axis. A Zemax analysis finds that the image of these LGSs will be affected by astigmatism and coma given in Table 5 . Other aberrations are insignificant. $\mathrm{Z}=85 \mathrm{~km}$ corresponds to the lowest expected position of the sodium layer at zenith. $\mathrm{Z}=150 \mathrm{~km}$ corresponds to the expected height of the sodium layer at $\sim 55$ degree zenith angle.

These aberrations will not significantly affect WFS spot images. However, each spot will be offset and the WFS will measure these aberrations. This aberrations are in the non-common path and should not be corrected by the DM. They can be removed by two different methods: 1) Correcting the aberrations optically before the WFS lenslet array or 2) Using the spot position corresponding to these aberrations as reference position and drive the spots to these positions (offset slopes).

The optical correction method is best from the standpoint of the AO control system, because it is transparent. Astigmatism does not depend on the LGS range distance and is therefore fairly easy to correct optically. Coma varies with range distance and therefore requires a moving corrector, which is much more involved.

The offset slopes correction method simplifies the opto-mechanical design, but suffers from two potential drawbacks: firstly, it uses a significant amount of dynamic range on the WFS: secondly, unavoidable calibration errors will make it so that only a fraction of the aberrations can be removed using this method. Experience with calibration of AO systems suggests that only a factor 3 to a factor 5 attenuation may be achieved. Table 6 also shows the offset slopes required to correct for each aberration, at the edge of the pupil, which is the worst case. These numbers are simply calculated by taking the derivatives of the corresponding Zernike polynomials. From the dynamic range standpoint, the WFS could fully correct these aberrations electronically using offset slopes. So we must now discuss the implications on the science image of only correcting these aberrations by a factor of 3 to 5 , because of calibration errors.

We focus our discussion on coma, because astigmatism is not a function of the range distance and can be easily corrected by a static corrector. Therefore we study the impact on the science image of leaving an increasing amount of coma. The results are given in Table 6 and show how much residual coma we can tolerate, how much coma can be corrected electronically, and how much should be corrected optically.

In Table 6 we find that the ITR would be reduced by $6 \%$ if no further electronic compensation is performed. With electronic compensation, the ITR reduction would likely be much less. Also, this is best seeing case. The ITR reduction would also be less in the median seeing case. We conclude that a static correction of coma is acceptable. Not including a static optical corrector for coma, leaving the full $1.78 \mu \mathrm{m}$ rms of coma going through would require electronic compensation at levels that we do not believe are reasonable. Our recommendation is therefore to include a static coma corrector in the LGS WFSs. 
Table 6. FWHM, EE50 and ITR of the seeing PSF, and the GLAO corrected PSF with an increasing amount of static coma added.

\begin{tabular}{|c|c|c|c|c|c|c|c|c|}
\hline & \multirow{2}{*}{$\begin{array}{l}\text { seeing } \\
\text { values }\end{array}$} & \multicolumn{7}{|c|}{ GLAO values with coma } \\
\hline & & $0 \mu m$ & $0.25 \mu m$ & $0.5 \mu \mathrm{m}$ & $0.75 \mu \mathrm{m}$ & $1.0 \mu \mathrm{m}$ & $1.25 \mu \mathrm{m}$ & $1.5 \mu \mathrm{m}$ \\
\hline FWHM(") & 0.18 & 0.15 & 0.15 & 0.16 & 0.17 & 0.18 & 0.19 & 0.21 \\
\hline $\operatorname{EE50}\left({ }^{\prime \prime}\right)$ & 0.29 & 0.21 & 0.22 & 0.23 & 0.25 & 0.27 & 0.29 & 0.30 \\
\hline ITR & 1.00 & 1.42 & 1.39 & 1.30 & 1.17 & 1.03 & 0.90 & 0.78 \\
\hline
\end{tabular}

\subsubsection{Expected residual spot jitter}

In GLAO, the LGS WFS sees a residual wave-front where the ground turbulence layer is corrected, but the rest of the turbulence is not. As a result, we can expect the WFS spots to be jittering nearly as much as when the loop is open. For the calculation we considered the tip-tilt of the uplink beam to be corrected. We also set the bottom layer of the TMT Science Requirements Document (SRD) to be perfectly corrected in the downlink. Using this turbulence profile with $r_{o}=15 \mathrm{~cm}$ (at $0.5 \mu \mathrm{m}$ ) and $L_{o} \rightarrow \infty$, the root mean square spot jitter is calculated to be no more than 22.2 or 25 milliarcseconds for a pitch of 1 or 0.5 meters. This residual jitter in the LGS spots will not seriously impact WFOS-GLAO performance.

\subsubsection{WFS linearity issues}

Centroiding error as a function of local tilt for an LGS WFS using radial format CCDs with 16x4 or 12x6 pixels per subaperture is studied in [8]. The linearity of the LGS WFS using a matched filter algorithm for optimal centroiding suggests that if a large offset is required (e.g. to correct for the static aberrations discussed in Section 4.2.2) then six pixels in the tangential direction is preferable (i.e. $12 \times 6)$. In this case a $(1,1)$ pixel offset appears to be possible. This corresponds to a 0.5 arcsecond offset which, based on the results in Table 5 would enable electronic correction of all coma.

\subsection{NGS WFS}

GLAO will use the On Instrument WFS (OIWFS) in each barrel for NGS WFS. Using only one NGS to sense tip-tilt for each $4.5^{\prime} \times 4.5^{\prime}$ sub-field should be sufficient for the GLAO PSF. Three of the WFOS barrels each have 2x2 Shack-Hartmann WFS and one barrel will likely have a higher order WFS to serve as the truth WFS. The two main purposes of the truth WFS 1) to monitor the true true atmospheric focus and thereby monitor the range to the sodium layer, which then controls the LGS WFS zoom optics and 2) monitor quasi-static aberrations at the science focal plane and correct for them by providing updated reference slopes to the LGS WFSs.

Our baseline is to use a $128 \times 128$ detector that can operate at 500 full frames per second. With $0.1^{\prime \prime}$ pixels this gives a 6.4 arcsecond field of view in each $64 \times 64$ pixel sub-aperture. It is desirable that at least one OIWFS has a larger field of view to make sure that the NGSs can be found even with telescope pointing errors and pick-off probe positioning errors. This avoids the overhead of pre-imaging the field. After the NGSs have been found, the telescope can be repointed and or the pick-off probes can be adjusted, until the NGSs are all centred in each WFS (see the operational model in Section 4.5).

Our baseline uses a 4 square arcminute patrol field that is inside the science field for each $2 \times 2$ OIWFS, introducing a small amount of vignetting for pick-off. As illustrated in [9] this field of view will be more than sufficient to give $100 \%$ sky coverage for sensing tip-tilt for WFOS-GLAO on TMT.A slightly larger patrol area would be desired for the truth WFS with $6 \times 6$ sub-apertures that also runs at 500 frames per second to monitor the sodium layer range. 


\subsection{Calibration sources}

WFOS does not have any entrance focal plane, so we are not considering outfitting the instrument with any source simulator that could be seen in the WFOS focal plane. There is the possibility of a deployable source in each of the LGS WFS arms. They would be just after the pick-off mirror / zoom corrector and just before the coma / astigmatism corrector. Such calibration sources could be used to:

- Measure the aberrations generated by the coma / astigmatism corrector and check that they are what they are supposed to be.

- Check pupil centring on the WFS lenslet array.

- Measure static aberrations that need to be considered as non-common path aberrations.

In order to access the WFOS focal plane, sources will have to be deployed at the TMT Prime focus. It would be useful to have just one LGS source that could feed an LGS WFS. Such a source would allow registration of the AM2 and WFSs, as well as test the sodium range compensation optics. Such an LGS source would not necessarily need to simulate the cone effect. However, it would need to simulate the finite range of the sodium layer, with the ability to vary this range. One LGS source would make it possible to close the AO loop on one LGS.

An additional NGS calibration source at the TMT Prime Focus would also be very desirable to monitor the image quality when the AO loop is closed on the LGS calibration source. It would need to be accessible from the truth WFS at least, and by all the other OIWFSs if possible.

\subsection{GLAO specific operations}

To make GLAO deployment have zero observational overhead we start the WFOS observations only with tip-tilt and focus correction using OIWFSs and the secondary mirror (OIWFS-M2 loop).

\section{Daytime perparation}

- Measure LGS WFS CCD noise level

- Deploy calibration source in each LGS WFS arm

- Check that the pupil is well centred on the lenslet arrays

- Check that the LGS WFS reads the correct coma correction

\section{Enabling GLAO correction}

- Open the laser shutters

- Adjust LGS positions (using LGSF steering mirrors) until the LGSs are properly centred on each LGS WFS.

- Check flux on LGS WFSs.

- Detune laser and record background image. This image will measure sky background, including Raleigh back-scattering and will be subtracted from the WFS measurements.

- Retune laser.

- Enable uplink T/T correction loop to stabilize LGS spots.

- Close high order GLAO loop using low loop gains to minimize transients that would perturb on-going science integration.

- Slowly ramp up the loop gains until the correction is optimal, while checking for system stability.

- The GLAO loop can remain closed during dithers or nods, as in nod-and-shuffle operation. 


\section{CONCLUDING STATEMENTS}

\subsection{Architecture trades summary}

Our preferred approach is to rely on the adaptive secondary mirror (AM2) being considered as the woofer for other TMT AO instruments. The gains predicted for all of the designs able to uniformly correct the full 81 square arcminutes are all characterized by modest integration time savings and as such are best operated as a transparent assist for seeing limited observations. If we consider the AM2 to be provided with one of the other TMT AO systems, the performance benefit of four single conjugate mirrors in each barrel (Arm) over the one AM2 designs is not considered enough. The AM2 woofer is compatible with WFOS-GLAO, is more elegant, correcting the wavefront before WFOS without crowding the spectrograph.

\subsection{Baseline system design summary}

The Natural Guide Star WFSs for GLAO would be the same OIWFSs needed for the seeing-limited mode. For LGS WFS at 8.5 arcminutes off axis we need coma corrector optics on each LGS WFS. The remainder of the aberrations can be corrected using offsets to LGS spots.

We find that a GLAO system would not introduce any additional overhead, including guide star acquisition, and is compatible with all the WFOS observing modes, including Nod-And-Shuffle. The WFOS-GLAO PSF will have a morphology similar to the seeing limited PSF, which means that GLAO can potentially be turned on or off during an exposure with no drastic consequence on the data quality, only a gain in efficiency when GLAO is turned on. This makes AM2 GLAO a very appealing add-on to WFOS.

\section{ACKNOWLEDGMENTS}

J.S. thanks Matthias Schoeck for his assistance with the T1 and T2 $C_{n}^{2}$ profiles. The authors gratefully acknowledge the support of the TMT partner institutions. They are the Association of Canadian Universities for Research in Astronomy (ACURA), the Association of Universities for Research in Astronomy (AURA), the California Institute of Technology and the University of California. This work was supported, as well, by the Canada Foundation for Innovation, the Gordon and Betty Moore Foundation, the National Optical Astronomy Observatory, which is operated by AURA under cooperative agreement with the National Science Foundation, the Ontario Ministry of Research and Innovation, and the National Research Council of Canada.

\section{REFERENCES}

1. J. Pazder, S. Roberts, R. Abraham, A. Anthony, M. Fletcher, T. Hardy, D. Loop, and S. Sun, "WFOS: A Wide Field Optical Spectrograph for the Thirty Meter Telescope," in these SPIE proceedings, 2006.

2. F. Rigaut, "Ground Conjugate Wide Field Adaptive Optics for the ELTs," in Beyond conventional adaptive optics, held May 7-10, 2001, Venice, Italy. Edited by E. Vernet, R. Ragazzoni, S. Esposito, and N. Hubin. Garching, Germany: European Southern Observatory, 2002 ESO Conference and Workshop Proceedings, 58, 2002.

3. A. Tokovinin, "Seeing Improvement with Ground-Layer Adaptive Optics," PASP 116, pp. 941-951, Oct. 2004.

4. M. Le Louarn and N. Hubin, "Improving the seeing with wide-field adaptive optics in the near-infrared," $m n$ 365, pp. 1324-1332, Feb. 2006.

5. J. Pazder, M. Fletcher, and C. Morbey, "The Optical Design of the Wide Field Optical Spectrograph for the Thirty Meter Telescope," in these SPIE proceedings, 2006.

6. L. Jolissaint, J.-P. Véran, and R. Conan, "Analytical modeling of adaptive optics: foundations of the phase spatial power spectrum approach," Optical Society of America Journal A 23, pp. 382-394, Feb. 2006.

7. J. Stoesz, "Constraining the GLAO parameter space with turbulence profile models," in these SPIE proceedings, 2006.

8. L. Gilles, B. Ellerbroek, and J.-P. Véran, "Laser guide star Shack-Hartman wavefront sensor modeling: matched filtering, wavefront sensor nonlinearity, and impact of sodium layer variability for the Thirty Meter Telescope," in these SPIE proceedings, 2006.

9. R. Clare, B. Ellerbroek, J.-P. Véran, G. Herriot, and D. Anderson, "Sky coverage and tip/tilt error analysis for the Thirty Meter Telescope," in these SPIE proceedings, 2006. 